\title{
Retraction Note to: Long non-coding RNA SNHG3, induced by IL-6/ STAT3 transactivation, promotes stem cell-like properties of gastric cancer cells by regulating the miR-3619-5p/ARL2 axis
}

\author{
Bo Sun ${ }^{1,2} \cdot$ Yang $\operatorname{Han}^{1,2} \cdot$ Hong Cai ${ }^{1,2} \cdot$ Hua Huang ${ }^{1,2} \cdot$ Yi Xuan $^{1,2}$ \\ Published online: 1 February 2022 \\ (c) Springer Nature Switzerland AG 2022
}

\section{Retraction Note to: Cellular Oncology (2021) 44:179-192 https://doi.org/10.1007/s13402-020-00560-2}

The Editors-in-Chief have retracted this article. After publication, cell image overlap was identified in Figs. 1c and $4 \mathrm{e}$, as well as among different panels in Fig. 4f. In addition, the SCG-7901 or BGC-823 cell lines, reported to be contaminated with HeLa cervical cancer cells, were used as a model of gastric cancer. The authors did not provide evidence of cell line validation. The Editors-in-Chief therefore no longer have confidence in the presented data and the conclusions of this article.

Authors Yang Han, Hong Cai and Yi Xuan agree to this retraction. Authors Bo Sun and Hua Huang have not responded to any correspondence from the editor or publisher about this retraction.

Publisher's Note Springer Nature remains neutral with regard to jurisdictional claims in published maps and institutional affiliations.

The original article can be found online at https://doi.org/10.1007/ s13402-020-00560-2.

Hong Cai

caihong450@hotmail.com

$\triangle$ Hua Huang

huahuang@fudan.edu.cn

$\checkmark$ Yi Xuan

xuanyi0118@126.com

1 Department of Gastric Surgery, Fudan University Shanghai Cancer Center, No. 270 Dongan Road, Shanghai 200032,

China

2 Department of Oncology, Shanghai Medical College, Fudan University, Shanghai 200032, China 